\title{
EDITORIAL
}

\section{The first step to unveil the epidemiology of inflammatory bowel disease in Central Asia}

\author{
Seung Wook Hong ${ }^{1}$, Byong Duk Ye ${ }^{1,2}$ \\ ${ }^{I}$ Department of Gastroenterology and ${ }^{2}$ Inflammatory Bowel Disease Center, Asan Medical Center, University of Ulsan College of Medicine, \\ Seoul, Korea
}

\section{Article: Prevalence and patient awareness of inflammatory bowel disease in Kazakhstan: a cross- sectional study (Intest Res 2020;18:430-437)}

Inflammatory bowel disease (IBD) is a disease in which the burden is rising worldwide. IBD is most commonly diagnosed in the second to fourth decade of life, and it impairs numerous aspects of an individual's life. ${ }^{2}$ Although the pathogenesis of IBD remains unclear, it is believed to be the consequence of an unregulated inflammatory response to the gut microbiome in a genetically susceptible host. ${ }^{3}$ Additionally, it is postulated that multiple environmental factors play significant roles in the development of IBD. ${ }^{3}$ In this aspect, epidemiological studies in various parts of the world could provide either direct or indirect insights into the impact of environmental factors in the development of IBD. Traditionally, IBD has been considered a common disease in high-income regions, such as North America, Western Europe, and Oceania. However, recent epidemiologic reports have shown a rapid rise in IBD incidence in the newly industrialized countries in Eastern Europe, Asia, and South America. ${ }^{4-7}$ Although the exact cause of the increase in IBD in previously low-incidence regions is still unclear, Westernization of society is believed to be associated with the accelerating incidence of IBD, making it a global disease. ${ }^{4}$ However, there have been no formal reports on the epi-

Received October 7, 2020. Accepted October 8, 2020.

Correspondence to Byong Duk Ye, Department of Gastroenterology and Inflammatory Bowel Disease Center, Asan Medical Center, University of Ulsan College of Medicine, 88 Olympic-ro 43-gil, Songpa-gu, Seoul 05505 Korea. Tel: +82-2-3010-3181, Fax: +82-2-476-0824,E-mail: bdye@amc. seoul.kr demiology of IBD in Central Asia, a large area in the middle of the Eurasian continent.

In the present issue of Intestinal Research, Kaibullayeva et al. ${ }^{8}$ first reported the prevalence of IBD in Kazakhstan. The authors enrolled a total of 115,556 subjects between January 2017 and December 2017 and confirmed 128 cases of IBD: 92 ulcerative colitis (UC) and 36 Crohn's disease (CD). Of the 128 patients, up to $20 \%$ (25 patients) were unaware of their diagnosis. The age- and sex-adjusted prevalence of IBD was 113.9 (69.0-158.9) per 100,000 population, including 84.4 (44.8123.9) for UC and 29.5 (8.2-50.9) for CD per 100,000 population. This estimated prevalence was much higher than that reported by the Ministry of Health of Kazakhstan (31.5 for UC and 6.3 for CD per 100,000 population). The authors presumed that the causes of these discrepancies arose from the differences in database systems and the accessibility to gastroenterological care. This study is noteworthy because the epidemiologic issues regarding IBD in Central Asia have rarely been addressed. Although this epidemiologic study could be positioned as the cornerstone of IBD epidemiological research in Central Asia, several issues should be considered when interpreting the results of this study.

First, the prevalence of IBD in this study was likely to be underestimated compared to the actual figure. Although the authors conducted a survey using questionnaires of more than 100,000 subjects from polyclinics, companies, and universities, the survey was limited to people aged 18 years and older, 
thereby possibly missing IBD patients in pediatric and adolescent age groups. Selection bias could also have been inherent in the study design by including only those who consented to the survey. Moreover, it is uncertain whether the distribution of age and sex in the survey respondents reflects the true population.

Second, after the survey, a confirmatory diagnosis of IBD was made sequentially by gastroenterological evaluation, fecal calprotectin test, and endoscopic and histologic evaluation. With this approach, savings in time and cost of the study could have been achieved. However, only a single test of fecal calprotectin and no incorporation of small bowel radiologic evaluation in the diagnostic algorithm might have missed true IBD patients with minimal to mild symptoms and patients with CD limited to the proximal small bowel.

Third, the authors compared the prevalence of IBD stratified into urban and rural areas, showing a numerically higher prevalence of both UC and CD in urban areas. However, there was a lack of socio-economic information to explain this regional discrepancy. For example, detailed information on the annual number of visits to healthcare facilities and household income by region could have disclosed the cause of urban-rural differences in IBD prevalence in more detail.

In conclusion, despite several limitations, this study has clinical significance because it is the first formal scientific report on the prevalence and characteristics of IBD in Central Asia. It also proposes a need to develop a strategy to enhance awareness of IBD in this region. Further studies on the evolving incidence of and risk factors for IBD in increasingly industrialized and urbanized societies are expected to provide novel insights into the etiopathogenesis of IBD.

\section{FINANCIAL SUPPORT}

The authors received no financial support for the research, authorship, and/or publication of this article.

\section{CONFLICT OF INTEREST}

No potential conflict of interest relevant to this article was reported.

\section{AUTHOR CONTRIBUTION}

Writing - original draft: Hong SW. Writing - review \& editing: Ye BD. Approval of final manuscript: all authors.

\section{ORCID}

Hong SW https://orcid.org/0000-0003-1440-9950

Ye BD https://orcid.org/0000-0001-6647-6325

\section{REFERENCES}

1. GBD 2017 Inflammatory Bowel Disease Collaborators. The global, regional, and national burden of inflammatory bowel disease in 195 countries and territories, 1990-2017: a systematic analysis for the Global Burden of Disease Study 2017. Lancet Gastroenterol Hepatol 2020;5:17-30.

2. Sairenji T, Collins KL, Evans DV. An update on inflammatory bowel disease. Prim Care 2017;44:673-692.

3. Baumgart DC, Carding SR. Inflammatory bowel disease: cause and immunobiology. Lancet 2007;369:1627-1640.

4. Ng SC, Shi HY, Hamidi N, et al. Worldwide incidence and prevalence of inflammatory bowel disease in the 21st century: a systematic review of population-based studies. Lancet 2018; 390:2769-2778.

5. Lakatos L, Mester G, Erdelyi Z, et al. Striking elevation in incidence and prevalence of inflammatory bowel disease in a province of western Hungary between 1977-2001. World J Gastroenterol 2004;10:404-409.

6. Park SH, Kim YJ, Rhee KH, et al. A 30-year trend analysis in the epidemiology of inflammatory bowel disease in the Songpa-Kangdong District of Seoul, Korea in 1986-2015. J Crohns Colitis 2019;13:1410-1417.

7. Kotze PG, Underwood FE, Damião AOMC, et al. Progression of inflammatory bowel diseases throughout Latin America and the Caribbean: a systematic review. Clin Gastroenterol Hepatol 2020;18:304-312.

8. Kaibullayeva J, Ualiyeva A, Oshibayeva A, Dushpanova A, Marshall JK. Prevalence and patient awareness of inflammatory bowel disease in Kazakhstan: a cross-sectional study. Intest Res 2020;18:430-437. 